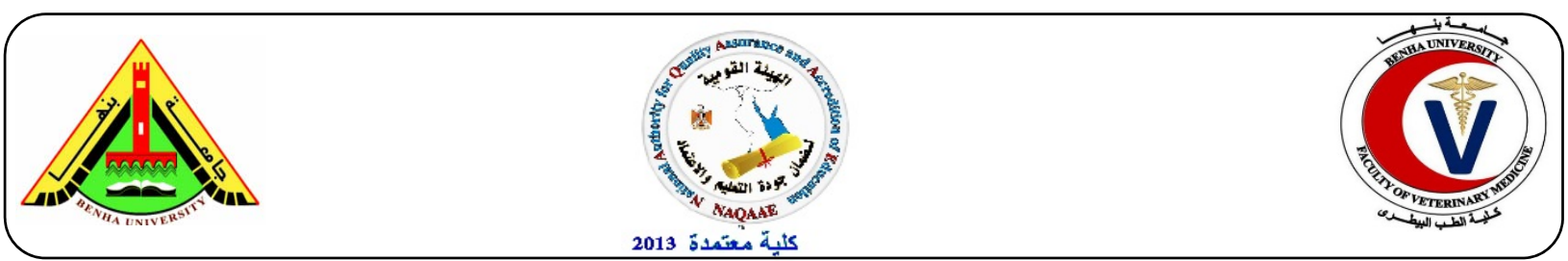

\title{
Study on Inactivated Equine herpesvirus-1 Vaccine Adjuvanted with Montanide
} Pet Gel A

\author{
Nehal S. Saleh \\ Veterinary Serum and vaccine Research Institute, Abbasia, Cairo, Egypt
}

\section{A B S T R A C T}

The study reported here is a part of a long-term research effort designed in our lab to select the best adjuvant, which can be used with Equine herpesvirus-1 (EHV-1) inactivated vaccine to induce good immune response in horses without any undesirable post vaccinal reaction. This study was carried out on Montanide pet gel A, which is an innovative adjuvant developed for companion animals. To evaluate the safety and potency of EHV-1 inactivated vaccine adjuvanted with Montanide pet gel A, It was inoculated into Guinea pigs as a preliminary evaluation, given mean complement fixing antibody titers of 67 and 176 at 21 and 35 days post inoculation (dpi) respectively, and mean ELISA antibody titers of 680 and 1322.5 were recorded at 21 and 35 dpi respectively, There was no any undesirable post vaccinal reaction in horses inoculated with the prepared vaccine. The immune response of the inoculated horses was monitored up to eight months post inoculation (mpi), Found that the peak of complement fixing and ELISA antibody mean titer was 213.3 and 1335 at 2.5 and 3 mpi respectively. ELISA antibodies persisted up to $6 \mathrm{mp}$. In conclusion, the prepared EHV-1 inactivated vaccine adjuvanted with montanide pet gel A is highly safe and potent for Horses.

Keywords: montanide pet gel, Equine herpesvirus-1, inactivated Vaccine

(http://www.bvmj.bu.edu.eg)

conference issue

(BVMJ-28(2): 26-33, 2015)

\section{1- INTRODUCTION}

$\mathrm{E}$ quine Herpes virus-1 (EHV-1) is one of the most common respiratory pathogens of the horses. EHV-1 induces several clinical signs of disease ranging in severity, from mild respiratory distress to abortion in pregnant mares, neonatal foal death and neuropathogenic disorders (Campbell and Studdert, 1983). Vaccination remains today one of the best options to fight EHV-1 infection in combination with management measures. Whole inactivated EHV-1 vaccines, which provide variable levels of protection against the disease through the induction of antibodies, have been the main type of vaccine commercially available. Successful vaccination against EHV-1 requires both humoral and cellular immune responses. Immunization with proteins (antigens) requires the presence of strong adjuvant to stimulate both immune responses (Paillot et al., 2008). Over the past 10 years many studies have attempted in Equine Vaccine Research Department at Veterinary Serum and Vaccine Research Institute (VSVRI) to characterize the safety and efficacy of different adjuvants used with EHV-1 inactivated vaccine e.g. DEAE-Dextran, Montanide ISA-70, ISA- 206, Mineral oil and Saponin (Nehal 2006, Safaa and Hussien, 2012, and Nehal et al., 2013). All adjuvants used gave acceptable protective humeral and cellular immune response, but most of them produce post vaccinal reaction at site of injection ranged from miled oedema to small or large sterile abscess. This reaction can be attributed to high sensitivity of horses, which need special adjuvant. Therefore, this study designed to evaluate the safety and potency of EHV-1 
inactivated vaccine adjuvanted with Montanide pet gel A, which an innovative adjuvant developed for companion animals (Dog, Cat and horses).

\section{2- Material and Methods}

\subsection{Virus}

Freeze-dried locally isolated EHV-1 at its VERO cell passage Two (VEp2) (Nehal et. al., 2009), was supplied by Equine vaccine Research Department, Veterinary Serum and Vaccine Research Institute (VSRI) and used for antigen and vaccine preparation.

\subsection{Antisera}

A) Reference freeze dried rabbit anti EHV1 Serum was kindly supplied by Dr. Jennet Wellington, Research follow, Dept. of biological science, Maquairia, Univ, NSW Australia and used for virus identity . B) Local Rabbit anti EHV-1 hyper immune serum prepared by Safaa et.al, (2005) was used as positive serum control in serological test.

\subsection{3- Animals}

\subsubsection{Horses}

Group of Five adult apparently healthy horses with low antibody titer against EHV1, OIE.2012. Three of them were used to evaluate the immunogenicity, potency and safety of the vaccine. The other two horses were kept as control.

\subsubsection{Guinea pig}

Group of 15 guinea pigs weighting approximately $350-450 \mathrm{~g}$ were used to evaluate immunogenicity of the prepared vaccine (Guo et al., 1989).

\subsubsection{Mice}

Two groups of pregnancy mice (10/ group), were used in safety test (OIE. 2012).

\subsection{Cell Culture}

African green monkey kidney cells (VERO) was maintained and grow in Eagl's minimum essential media supplemented with $10 \%$ newly born calf serum for growth media only, and penicillin sodium $100 \mathrm{IU} / \mathrm{ml}$. with streptomycin $100 \mathrm{mg} / \mathrm{ml}$. It was used for virus propagation.

\subsection{Binary Ethyleneimine}

0.1M Binary Ethyleneimine stock solution prepared from 2-bromoethylamine hydrobromide (Aldrich chemical Co., LTD) and $0.2 \mathrm{~N} \mathrm{NaOH}$, according to Bahnemann 1990 and Mark 2004, was used for EHV-1 inactivation process.

\subsection{Montanide pet gel A}

It is an innovative, ready-to-disperse polymeric adjuvant designed to improve the safety and efficacy of vaccines for companion animals. It is a sterile adjuvant manufactured by Seppic Company. Used in ratio of $10 \%$.

\subsection{Preparation of $E H V-1$ vaccine}

A master seed EHV-1(local isolate VEp2) was grown on VERO cells for another three passage to prepare vaccine seed virus (VEp5), which subjected to identity test. From the vaccine seed virus, a vaccine stock viral fluid was prepared by caring another one passage on VERO cell and the bulk virus harvest fluids was kept at -70 tell the titration and sterility test was done. Vaccine stock viral fluid having virus titer not less than $7 \log _{10}$ TCID $50 / m l$ (Mayer et al. , 1978) was inactivated by $0.008 \mathrm{M} \mathrm{BEI}$ at $37^{\circ} \mathrm{C}$ for 24 hours (Nehal, 2006). Finally the montanide pet gel A (adjuvant) was add in ratio of $10 \%$, into the inactivated virus fluid while mixing on a magnetic stirrer in a low speed at room temperature tell ensure perfect homogeneity of the complete batch, according to manufacture direction, Seppic Company. The prepared EHV-1vaccine distributed in vials contained one dose $(2 \mathrm{ml}$ /dose), caped and kept at $4^{\circ} \mathrm{C}$.

\subsection{Identity test}

Was done by SNT according to OIE, (2012) using reference anti sera against EHV-1.

\subsection{Sterility Test}

Samples were taken from the final product as well as the virus fluid before inactivation 
process and tested on Nutrient agar medium, Sabouraud dextrose agar medium, Thioglycolate medium (Oxford, England) and PPLO (broth) medium for bacterial fungal and mycoplasma contaminations (OIE. 2012).

\subsection{Safety Test}

It was performed according to OIE. (2012). The residual infective virus activity was examined by inoculation of inactivated virus fluid on VERO cells which was incubated at $37^{\circ} \mathrm{C}$ for seven days with daily observation. Additionally blind passage was done to ensure complete virus inactivation. Group of mice at late stage of pregnancy were inoculated intranasal (I/N) by $45 \mu \mathrm{l}$ of inactivated virus fluid. Another group of mice at lat stage of pregnancy were inoculated subcutaneous $(\mathrm{S} / \mathrm{C})$ by two dose from the final product with one week interval $(0.2 \mathrm{ml} /$ dose $)$.

All experimental mice were kept under observation until parturition.

\subsection{Immunization of guinea pig}

In order to determine the immunogenicity of the prepared vaccine, a Group of Ten guinea pigs received (2 $\mathrm{ml} /$ dose $)$ subcutaneously $(\mathrm{S} / \mathrm{C})$ from the prepared vaccine, followed by a booster dose on the day 21. Another Five guinea pigs were kept as control. The guinea pigs were bled before the primary inoculation and on days 21 and 35 (Guo et. al., 1989). Sera were prepared and tested for the presence of EHV-1 antibodies by CFT and ELISA.

\subsection{Immunization of horse}

Three horses were inoculated I/M with the prepared vaccine $(2 \mathrm{ml} /$ dose $)$ as an initial dose, followed by a booster dose at 6 weak and $2^{\text {nd }}$ dose at 6 month. Body temperature of the immunized horses was measured and the sites of inoculation were observed daily for seven days post each inoculation for detection of any undesirable post vaccinal reaction. Serum samples were collected from all horses at intervals to monitor the immunization curve.

\subsection{Serological tests}

\subsubsection{Enzyme linked immunosorbent assay (Solid phase ELISA):}

Indirect ELISA (Single-dilution) was carried according to Crabb and Studdert, (1993) and Sugiura et al., (1997). It was done to monitoring the immune response of inoculated Guinea pigs and horses; the end titer of the tested sample was calculated according to Williams (1987) as follow:

OD of tested sample-OD of negative control $\mathrm{X}$ titer of positive control

OD of positive control - OD of negative control

\subsubsection{Complement Fixation test (CFT):}

The CFT using polyethylene glycol (PEG) concentrated EHV-1 antigen was performed (Singh, 2002).on sera collected from inoculated guinea pigs and horses. CF antibodies titer was expressed as the reciprocal of the highest serum dilution reducing the RBCs haemolysis to $50 \%$ or less.

\section{3- RESULTS}

\subsection{Identity test}

Vaccine seed virus (VEps) was completely neutralizing $(100 \%)$ by reference anti sera against EHV-1.

\subsection{Virus titration}

The infectivity titer of the vaccine viral fluid ( EHV-1 VEp6) was $7 \log _{10}$ TCID $_{50} / \mathrm{ml}(7.3$ $\log _{10} \mathrm{TCID}_{50} / 2 \mathrm{ml} /$ dose).

\subsection{Sterility Test}

The Vaccine viral fluid as well as the final vaccine products proved to be sterile without any bacterial, fungal and mycoplasma contaminations when tested on special bacterial and fungal media.

\subsection{Safety Test}

Neither CPE in two successive blind passages on VERO cells (inoculated with the inactivated virus fluid) nor abortion or untoward reaction (roughness, loss of 
weight, nervous signs, death, hypersensitivity) were detected in pregnant mice inoculated with inactivated virus or with the final vaccine product.

\subsection{Immunization of guinea pig}

EHV-1 antibodies were detectable in inoculated guinea pig 21 days after the primary inoculation with mean complement fixing antibodies and ELISA titer 64 and 680 respectively. A significant increase in the antibody titer (about 2 fold) was obtained by 2 weeks after a second inoculation of the vaccine (at $35 \mathrm{dpi}$ ) with mean CF antibodies and ELISA titer 176 and 1322.5 respectively as shown in (table $1 \& 2$ and Fig. 1$)$

\subsection{Immunization of horse}

In the vaccinated horses the mean $\mathrm{CF}$ antibodies titer as shown in (table 3 and Fig.2), were increased 4- fold within the first four weeks post inoculation (wpi) of initial dose (from 10.7 at 2 wpi to 42.67 at 4wpi).The peak of $\mathrm{CF}$ antibodies was regarded at 1 and 1.5 months post both booster and $2^{\text {nd }}$ dose with mean titers 213.3 and 341.3 respectively. Positive protective CF antibody titers $\geq 20$ can be detected for 2.5 months post booster dose. While the ELISA result as shown in (table 4 and Fig. 3 ) the first dose of the prepared vaccine was able to stimulate reasonable antibody response detected at 2 wpi with a mean ELISA titer 450 and reached its peak at the 4wpi with a mean ELISA titer 923.3. By booster and 2nd dose a much higher level of antibodies was developed, reached their maximum at the 1.5 and 2 months post each dose with a mean value 1335 and 2370 respectively. Then the ELISA antibodies titer began to decline gradually till 6th month post vaccination, with a considerable protective antibody level.

Table (1): EHV-1 CF antibody titers in inoculated Guinea pigs.

\begin{tabular}{ccc}
\hline Time of sampling & Mean antibody titer & Non inoculated control \\
\hline 0Time & - ve & $-v e$ \\
& $1^{\text {st }}$ inoculation & \\
21dpi* & $64^{* *}$ & $-v e$ \\
35dpi & Booster dose & $-v e$ \\
\hline
\end{tabular}

*dpi: days post inoculation. ${ }^{* *} \mathrm{CF}$ antibody titer= Reciprocal of the highest dilution of serum showed positive result.

Table (2): EHV-1 ELISA titers in inoculated Guinea pigs

\begin{tabular}{ccc}
\hline Time of sampling & Mean antibody titer & Non inoculated Control \\
\hline 0Time & -ve & -ve \\
& $1^{\text {st }}$ inoculation & \\
$21 \mathrm{dpi}^{*}$ & $680^{* *}$ & $-\mathrm{ve}$ \\
$35 \mathrm{dpi}$ & Booster dose & -ve
\end{tabular}

*dpi: days post inoculation. **ELISA titer= Reciprocal of the highest dilution of serum showed positive result. 
Fig. (1): EHV-1 CF antibodies and ELISA titer in inoculated Guinea pigs

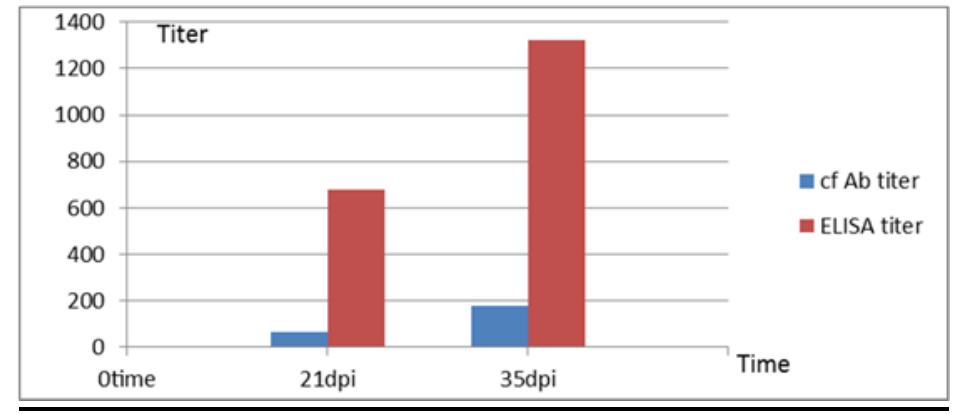

Dpi: days post inoculation

Table (3): EHV-1CF antibody titers in inoculated horses

\begin{tabular}{|c|c|c|c|c|c|}
\hline \multirow{3}{*}{ Time of sampling } & \multicolumn{5}{|c|}{ EHV-1 CF antibody titer } \\
\hline & \multicolumn{3}{|c|}{ Inoculated horses } & \multirow[t]{2}{*}{ Mean } & \multirow{2}{*}{$\begin{array}{c}\text { Non inoculated } \\
\text { Control }\end{array}$} \\
\hline & $\mathrm{H} 1 *$ & $\mathrm{H} 2$ & $\mathrm{H} 3$ & & \\
\hline \multirow{2}{*}{0 Time } & $-\mathrm{ve}$ & -ve & $--\mathrm{ve}$ & - & 0 \\
\hline & \multicolumn{3}{|c|}{ Inoculation of initial dose } & & \\
\hline 2wpi & $8 * *$ & 16 & 8 & 10.7 & 0 \\
\hline 4wpi & 32 & 64 & 32 & 42.67 & 0 \\
\hline \multirow[t]{2}{*}{ 6wpi } & 16 & 32 & 8 & 18.7 & 0 \\
\hline & \multicolumn{3}{|c|}{ Booster dose } & & \\
\hline $2 \mathrm{mpi}$ & 64 & 128 & 64 & 85.3 & 0 \\
\hline $2.5 \mathrm{mpi}$ & 128 & 256 & 256 & 213.3 & \\
\hline $3 \mathrm{mpi}$ & 64 & 128 & 128 & 106.7 & 0 \\
\hline $4 \mathrm{mpi}$ & 16 & 32 & 16 & 21.3 & 0 \\
\hline $5 \mathrm{mpi}$ & -ve & 4 & 4 & 2.7 & 0 \\
\hline \multirow{2}{*}{$6 \mathrm{mpi}$} & -ve & -ve & -ve & - & 0 \\
\hline & \multicolumn{3}{|c|}{ 2nd dose } & & \\
\hline $6.5 \mathrm{mpi}$ & 32 & 64 & 32 & 42.7 & \\
\hline $7 \mathrm{mpi}$ & 128 & 256 & 128 & 170.7 & 0 \\
\hline $7.5 \mathrm{mpi}$ & 256 & 512 & 256 & 341.3 & 0 \\
\hline $8 \mathrm{mpi}$ & 128 & 128 & 256 & 170.7 & 0 \\
\hline
\end{tabular}

Fig.(2):EHV-1 CF antibody titers in inoculated Horses

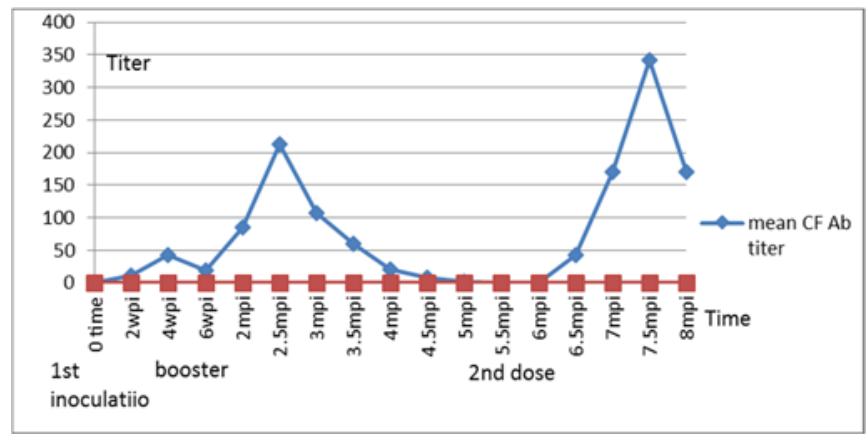


Table (4): EHV-1 ELISA antibody titers in inoculated horses

\begin{tabular}{|c|c|c|c|c|c|}
\hline \multirow{3}{*}{$\begin{array}{r}\text { Time of } \\
\text { sampling }\end{array}$} & \multicolumn{5}{|c|}{ EHV-1 ELISA titer } \\
\hline & \multicolumn{3}{|c|}{ Inoculated horses } & \multirow{2}{*}{ Mean } & \multirow{2}{*}{$\begin{array}{c}\text { Non inoculated } \\
\text { Control }\end{array}$} \\
\hline & $\mathrm{H} 1 *$ & $\mathrm{H} 2$ & $\mathrm{H} 3$ & & \\
\hline \multirow{2}{*}{ 0Time } & $165^{* *}$ & 170 & 150 & 161.7 & 170 \\
\hline & \multicolumn{3}{|c|}{ Inoculation of initial dose } & & \\
\hline 2wpi & 430 & 480 & 440 & 450 & 160 \\
\hline 4wpi & 900 & 950 & 920 & 923.3 & 160 \\
\hline \multirow[t]{2}{*}{ 6wpi } & 700 & 810 & 880 & 796 & 170 \\
\hline & \multicolumn{3}{|c|}{ Booster dose } & & \\
\hline $2 \mathrm{mpi}$ & 950 & 1050 & 1000 & 1000 & 160 \\
\hline $2.5 \mathrm{mpi}$ & 1070 & 1230 & 1300 & 1200 & 160 \\
\hline $3 \mathrm{mpi}$ & 1265 & 1450 & 1290 & 1335 & 160 \\
\hline $4 \mathrm{mpi}$ & 1000 & 1200 & 1160 & 1120 & 160 \\
\hline $5 \mathrm{mpi}$ & 820 & 900 & 960 & 893.3 & 170 \\
\hline \multirow{2}{*}{$6 \mathrm{mpi}$} & 650 & 700 & 630 & 660 & 160 \\
\hline & \multicolumn{3}{|c|}{ 2nd dose } & & \\
\hline $6.5 \mathrm{mpi}$ & 1300 & 1750 & 1540 & 1530 & 160 \\
\hline $7 \mathrm{mpi}$ & 2000 & 2570 & 2300 & 2290 & 160 \\
\hline $8 \mathrm{mpi}$ & 2020 & 2530 & 2560 & 2370 & 160 \\
\hline
\end{tabular}

wpi : week post inoculation mpi : month post inoculation * Horse number

**ELISA titer $=$ Reciprocal of the highest dilution of serum showed positive result.

Fig. (3): EHV-1 ELISA titer in inoculated Horses

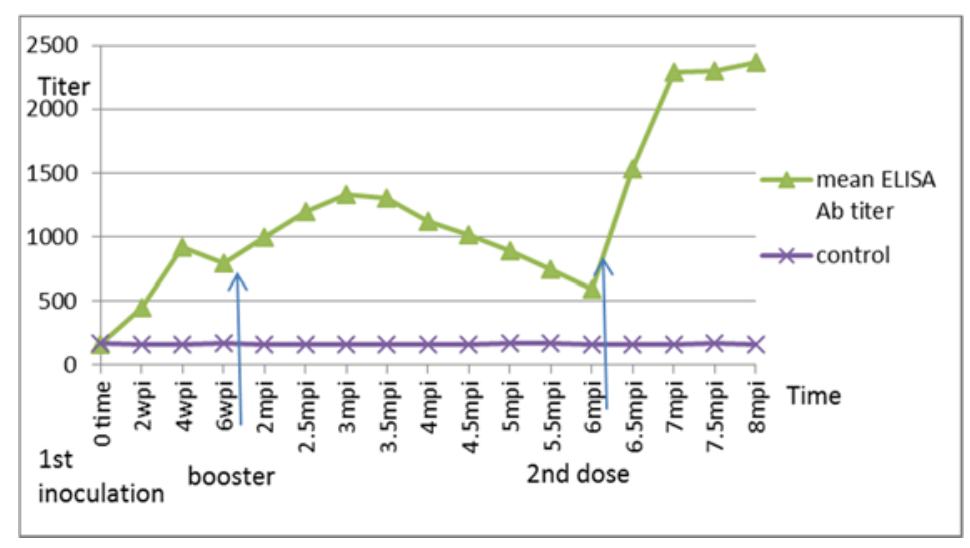

\section{4- DISCUSSION}

Successful vaccination against EHV-1 with inactivated vaccine require presence of strong adjuvant to maximize the vaccine potency, on the other hand horse are highly valuable sensitive animals which need special adjuvant, that don't produce undesirable post vaccinal reaction either locally nor systemic. This paper studies the safety and potency of EHV-1 inactivated vaccine adjuvanted with Montanide pet gel $\mathrm{A}$ in horses and guinea pigs. To prepare this vaccine, the EHV-1(vaccine seed virus) was subjected to identity test by using SNT (OIE, 2012) then propagated on VERO cells and titrated, The infectivity titer of the EHV-1 VEp6 (vaccine viral fluid) was 7 $\log _{10} \quad \mathrm{TCID}_{50} \quad / \mathrm{ml} \quad\left(\begin{array}{llll}7.3 & \log _{10} & \mathrm{TCID}_{50}\end{array}\right.$ $/ 2 \mathrm{ml} /$ dose. This titer exceeds the 
immunizing dose $\left(6.1 \log _{10}\right.$ TCID $\left.50 / 2 \mathrm{ml}\right)$ that reported by Charles, et. al., (1977). Vaccine viral fluid has been completely inactivated by $0.008 \mathrm{M} \mathrm{BEI}$ at $37^{\circ} \mathrm{C}$ for 24 hours (Nehal, 2006). The immunogenicity of the prepared vaccine was pre-estimated in guinea pig elicited an obvious immune response (table $1 \& 2$ and Fig.1). EHV-1 antibodies were detectable 21 days after the primary inoculation with mean complement fixing antibodies and ELISA titer 64 and 680 respectively. A significant increase in the antibody titer (about 2 fold) was obtained by 2 weeks after a second inoculation of the vaccine (at 35 dpi) with mean CF antibodies and ELISA titer 176 and 1322.5 respectively, This result was similar to the result recorded by Guo et al., (1989) and revealed that the prepared vaccine is immunogenic. Respecting to horses immunization there was no detectable adverse reaction observed in any horses after inoculation of the prepared vaccine. The immune response of the inoculated horses was monitored up to eight months post inoculation (mpi).It was found that the mean $\mathrm{CF}$ antibodies titer as shown in (table 3 and Fig.2), were increased 4- fold within the first four weeks post inoculation (wpi) of initial dose (from 10.7 at 2 wpi to 42.67 at 4 wpi). The peak of CF antibodies was regarded at 1 and 1.5 months post both booster and $2^{\text {nd }}$ dose with mean titers 213.3 and 341.3 respectively. Positive protective CF antibody titers $\geq 20$ can be detected for 2.5 months post booster dose. These results come parallel to results obtained by Burrows et al., (1984) and Singh et al.,(2006), who stated that CF antibodies titer increased 2 to 4 fold following each injection of the vaccine and can be detected for 2 to 3 months, Although horses with $\mathrm{CF}$ antibodies titer 20 showed reduction in the incidence and duration of clinical signs after challenge. In concerning to the ELISA result as dedicated in (table 4) and illustrated in (Fig. 3) the first dose of the prepared vaccine was able to stimulate reasonable antibody response detected at 2 wpi with a mean ELISA titer 450 and reached its peak at the 4 wpi with a mean ELISA titer 923.3. By booster and 2nd dose a much higher level of antibodies was developed, reached their maximum at the 1.5 and 2 months post each dose with a mean value 1335 and 2370 respectively. Then the ELISA antibodies titer began to decline gradually till 6th month post vaccination, with a considerable protective antibody level. These results agree with Bannai et al., (2014) and Rusli et al., (2014) who found that ELISA antibodies begin to increase by day 14 post injection then reached its peak at 2 months with 4 fold increasing which indicate good immune response. In conclusion, the prepared EHV1 inactivated vaccine adjuvanted with montanide pet gel A is highly safe and potent for Horses.

\section{5- REFERENCES}

Bahnemann, H.G. 1990. Inactivation of viral antigens for vaccine preparation with particular reference to the application of binary ethyleneimine vaccine, 8: 299303.

Bannai, H.; Mae, N.; Ode, H.; Nemoto, M.; Tsujimura, K.; Yamanaka, T.; Kondo, T. and Matsumura, T. 2014. Successful control of winter pyrexias caused by EHV-1 in Japanese training centers by achieving high vaccination coverage. Clin. Vaccine Immunology. 21(8):10701076.

Burrows, R.; Goodridge, D. and Denyer, M. S. 1984. Trials of an inactivated equid herpesvirus-1 vaccine: Challenge with a subtype 1 virus. Veterinary Record, 14: 369- 374.

Campbell, T.M. and Studdert 1983. EHV type 1. Vet. Bull., 53(2):135-147.

Charles, W.; Purdy; Shirley, J. Ford and William, F. Grant 1977. Equine Rhinopneumonitis Virus (Herpesvirus Type 1) Attenuation in Stable Monkey Cell Line. Am. J. Vet. Res, 38(8):12111215.

Crabb, S. Brendan and Studdert, J. Michael. 1993. Epitopes of glycoprotein $G$ of Equine Herpesviruses 4 and 1 located near the $\mathrm{C}$ termini elicit type-specific 
antibody responses in the natural host. J. of Virology 67(10):6332-6338.

Guo Peixuan, Scott Goebel, Stephen Davis, Marion E. Perkus, Bernard Languet, Philippe Desmetter, George Allen and Enzo Paoletti 1989. Expression in Recombinant Vaccinia Virus of the Equine Herpesvirus 1 Gene Encoding Glycoprotein gpl3 and Protection of Immunized Animals. J. Virol., 63(10):4189-4198.

Mark, W. Mellencamp 2004. Chemically inactivated ehv-1 kya virus; and an adjuvant which includes cross-linked olefinically unsaturated carboxylic acid polymer. Patents, publication number US $6803041 \mathrm{~B} 2$.

Mayr A., Thein, P. \& Scheid, N. 1978. Immunization experiments with inactivated EHV-1. In proceeding $4^{\text {th }}$ Int. Conf., Equine infect. Dis., Lyon edited by Bryans, J.T. and Gerber Vet. Pub. Inc. princtone, New Jercy, PP. 57-67, 1975.

Nehal, S. Saleh, 2006. Preliminary trials for production of equine viral abortion inactivated vaccine. $\mathrm{Ph}$. D. Thesis, Virology. Benha Univ.

Nehal,S. Saleh; Eman, M. Ebied; Magda, A. Kalad and Solima I.M.A. (2009):Recent isolation and identification of equine herpesvirus abortion (EHV-1)in Egypt 2007. Vet. Med. J. 57(4):800-820.

Nehal, S. Saleh; Safaa, A. Warda; Eman, M. Ebeid; Nashwa, K. Madkour \& Magda, A. Kalad, 2013. Monitoring the immune response of horse vaccinated with inactivated Equine herpes virus-1 vaccine Ippologia, Anno 24, 3-4, Settembre-Dicembre.17-23.
OIE 2012. Equine Rhinopneumonitis. Terrestrial Manual $7^{\text {th }}$ edition chapter 25-9 pp, 889-898.

Paillot, R.; Case R.; Ross J.; Newton R. and Nugent J. 2008. Equine Herpes Virus-1: Virus, Immunity and Vaccines. The Open Veterinary Science Journal, 268291.

Rusli, N. Dini; Mat, K. Binti; Harun, H. Che. 2014. A Review: Interactions of Equine Herpesvirus-1 with Immune System and Equine Lymphocyte Open Journal of Veterinary Medicine, Vol. 4, p. 294-307

Safaa, A. Warda, and Hussien G.M. 2012. Immune response of horses vaccinated with inactivated oil (Montanide ISA-70) adjuvanted EHV-1vaccine. Egypt, J. Agrc. Res., 90(3):1309-1321.

Safaa, A. Warda; Taradi, Said and Daoud, A.M. 2005. Trials for preparation and evaluation of locally prepared polyclonal immune serum against EHV-1. $4^{\text {th }}$ Int. Sci.

Singh, B.K., 2002. Immunological response of ponies to an inactivated Indian strain of equine herpesvirus-1, Indian J. Anim. Sci., 72:831- 835 .

Singh, B.K.; Tandon, S. N. and Virman, N. 2006. Immune response to oil inactivated Equine herpesvirus- 1using different emulsifiers in horses. Indian J. Biotec., 5:42- 46 .

Sugiura, T.; Kondo, T.; Matsumura, T.; Imagawa, H.; Kamada, M. and Ihara, T. 1997. Evaluation of enzyme linked immunosorbent assay for titration of antibodies to EHV-1. J. Equine Sci., 8(3):57-61.

Williams, R. 1987. ELISA technique for diagnosis of AHSV.J. Vet. Diag. Invest., 11(2):9-11. 